\title{
Petra Schaper-Rinkel
}

\section{Governance von Zukunftsversprechen: Zur politischen Ökonomie der Nanotechnologie}

\section{Einleitung $^{1}$}

Nanotechnologie gilt als Schlüsseltechnologie des 21. Jahrhunderts, als eine Querschnitttechnologie, die wie die Informationstechnologie des 20. Jahrhunderts allgegenwärtig sein wird. Heute werden Nanopartikel als Schutzschichten für Verglasungen und Brillenoberflächen eingesetzt, UV-absorbierende Nanopartikel sind in Sonnencremes und Sonnenschutztextilien enthalten, Elektronik wird durch Nanotechnologie optimiert; in der Zukunft sollen mit ihr selbstreinigende Fenster, Bibliotheken am Handgelenk und Prothesen aus biokompatiblen Stoffen möglich sein (BMBF 2004a; BMBF 2005). Was die Nanotechnologie konzeptionell zu einer Schlüsseltechnologie macht, ist das Versprechen auf eine neue industrielle Revolution in der Zukunft: Die Handlungsfähigkeit auf atomarer und molekularer Ebene stellt konzeptionell eine praktische Weltformel dar, verbunden mit der Vorstellung, Alles 'Atom für Atom' in gewünschter Weise aufbauen zu können (National Science and Technology Council 1999). Mittlerweile wird Nanotechnologie weltweit mit jährlich ungefähr 4 Mrd. Euro an öffentlichen Mitteln gefördert (Luther/Bachmann et al. 2006: 9).

Die radikalste Vorstellung einer zukünftigen Nanotechnologie wurde schon in den achtziger Jahren unter dem Stichwort „molecular manufacturing“ (Molekulare Fertigung) von dem US-amerikanischen Physiker Eric Drexler entwikkelt (Drexler 1987). „Die digitale Revolution hat sich auf ein Gerät konzentriert, durch das sich fast jedes gewünschte Bit-Muster erzeugen lässt: den programmierbaren Computer. Genauso wird die mit der Nanotechnologie einhergehende Revolution um eine Vorrichtung kreisen, mit der sich fast jede gewünschte Anordnung von Atomen herstellen lässt: den programmierbaren Assembler." (Drexler/Peterson et al. 1991: 35). Zwar gilt die Realisierung von Drexlers Vorstellungen als ausgesprochen unwahrscheinlich, doch findet sich das Konzept in vorsichtigerer Form in technologiepolitischen Programmen (vgl. Milburn 2002; Nordmann 2003; TAB 2004: 145ff) und bildet das große

1 Dieser Artikel beruht auf empirischen Arbeiten im Rahmen eines von der DFG geförderten Projekts an der Freien Universität Berlin. Für weitere Informationen siehe: www.nanotechnologiepolitik.de.

PROKLA. Zeitschrift für kritische Sozialwissenschaft, Heft 145, 36. Jg., 2006, Nr. 4, 473-496 
Versprechen im Hintergrund alltäglicherer Technologiepolitik: Dezentral würde jeder alles Lebensnotwendige produzieren, ohne abfallproduzierende Fabriken, auf der Basis von Solarenergie und ohne Nutzung fossiler Energieträger. Damit wäre theoretisch die kapitalistische Produktionsweise abgeschafft, schließlich wären die Produktionsmittel in der Hand Aller und so würden nur noch Gebrauchswerte erzeugt werden. Allerdings gehen weder die radikalen noch die pragmatischen Verfechter der Nanotechnologie von einer Abschaffung des Kapitalismus aus, oder würden diese propagieren. Vielmehr soll die Nanotechnologie ungeahnte Wachstumsmärkte schaffen und die Wettbewerbsfähigkeit traditioneller Industrien erhöhen. Dies ist nur eine der zahlreichen Paradoxien, die mit der Nanotechnologie verknüpft sind. Eine andere besteht darin, dass in der staatlichen Nanotechnologiepolitik zwar die Chancen gebetsmühlenartig wiederholt werden, die Risiken aber lange tabuisiert wurden. So hatte Drexler bereits von vornherein auf die symmetrische Zerstörungskraft unkontrollierbarer Nanomaschinen (das sogenannte Grey-Goo Phänomen) verwiesen; ein Gedanke, den der US-amerikanische Computer-Experte und Chief Scientist von Sun Microsystems, Bill Joy, im Jahr 2000 in einem Aufsehen erregenden Zeitungsartikel zum Ausgangspunkt für die Warnung vor den weltbedrohenden Gefahren ${ }^{2}$ von Gentechnik, Nanotechnologie und Robotik nahm (Joy 2000a; Joy 2000b). Der Bestsellerautor Michael Crichton hat ihn zu einem Roman verarbeitet, in dem die Menschheit von sich selbst replizierenden Nanobots bedroht wird (Crichton 2002). ${ }^{3}$ Diese rein hypothetischen Risiken einer molekularen Nanotechnologie haben ein hohes $\mathrm{Maß}$ an Öffentlichkeit auf sich gezogen, in deren Gefolge nun auch die Risiken der kurz- und mittelfristigen Anwendungen zunehmend Beachtung finden (BMU/Umweltbundesamt et al. 2005). Denn die spezifischen Eigenschaften, die nanoskalige Partikel haben, und die zukünftig so gewinnbringend verwertet werden sollen ${ }^{4}$, sind in ihren langfristigen Nebenfolgen wenig absehbar (Swiss Re 2004a:40).

2 „Nanoboter besitzen eine gefährliche Eigenschaft: Sie können sich selbständig vermehren. Eine Bombe explodiert nur einmal, aus einem einzigen Roboter können viele werden, die rasch außer Kontrolle geraten " (Joy 2000a).

3 Crichtons Roman ist die einzige Nano-Science-Fiction, die von Meinungsforschung und Akteuren der Nanotechnologiepolitik umfassend zur Kenntnis genommen wird. Aufgrund der weltweiten Filmerfolge seiner Bücher (,Jurassic Park“) wurde in der Verfilmung des Buches (die es entgegen vieler Ankündigungen bis heute nicht gibt) eine Bedrohung für die Akzeptanz der Nanotechnologie gesehen. Umfassende Nano-Szenarien sind in Neil Stephensons „Diamond Age. Die Grenzwelt“ (Stephenson 1996) und in John Robert Marlows „Nano“ (Marlow 2005) zu finden.

4 Zum Beispiel sind Nanopartikel in der Lage, die ansonsten schwer überwindbare Blut-HirnSchranke zu durchbrechen und können gezielt Medikamente in spezifische Zellen - wie Krebszellen - schleusen. Damit werden neue Therapien für Gehirn- und Krebserkrankungen möglich. Zugleich können aber vielleicht auch andere Nanopartikel diese Schranke überwinden und zerstörerisch wirken. Und außerdem lassen sich solche Nanopartikel nicht nur als Träger für Medikamente verwenden, sondern könnten in militärischen Anwendungen genauso als Trägersubstanz für neuartige lähmende oder tödliche Substanzen genutzt werden. 
Dies wiederum ist ein Phänomen, das sich im Lichte der Erfahrungen des 20. Jahrhunderts als fatal erweisen könnte: Krebs durch Asbest und die Zerstörung der Ozonschicht durch FCKW sind nur zwei Beispiele für die langfristigen Schäden, die aus einstmals gefeierten technischen Innovationen entstanden sind (EEA 2001). Ausgehend von den Versprechen und Widersprüchen dreht sich die Beherrschung und Regierung des Nanokosmos um drei Fragekomplexe: Was sind die Grenzen und Möglichkeiten der Nanotechnologie? Welche daraus resultierenden Anwendungen sollen forciert werden? Und wie lässt sich eine sichere und verantwortungsvolle Entwicklung der Nanotechnologie gewährleisten?

\section{Zur Geschichte der Nanotechnologie: Was treibt die Entwicklung an?}

Wie konstituiert sich ein technologisches Feld und wie erklärt es sich, dass die Nanotechnologie als Schlüsseltechnologie des 21. Jahrhunderts gilt? Diese Geschichte lässt sich traditionell als lineare wissenschaftlich-technische Fortschrittsgeschichte auffassen; bei näherer Betrachtung zeigt sich jedoch, dass die Dynamik der Nanotechnologie stark technologiepolitisch bestimmt ist. Am Anfang - so die Standarderzählung der Nanotechnologie (vgl. kritisch hierzu: Milburn 2002; Baird/Shew 2004) - stehen Konzept und Vision. Am 29. Dezember 1959 formulierte der amerikanische Physiker und spätere Nobelpreisträger Richard Feynman auf dem jährlichen Treffen der Amerikanischen Physikalischen Gesellschaft am California Institute of Technology (Caltech) den konzeptionellen Paradigmenwechsel der Miniaturisierung. Unter dem Titel „There's Plenty of Room at the Bottom“, sprach er von der Idee, statt wie bisher Dinge nur zu verkleinern, sie in ferner Zukunft auch aus den kleinsten Teilchen konstruieren zu können (Feynman 1959). Feynman kam in seinen Forschungen nicht auf diese Idee zurück, sein Gedanke wurde seinerzeit nicht aufgegriffen, noch existierte der Begriff der Nanotechnologie. Diesen benutzte erst 1974 der japanische Wissenschaftler Norio Taniguchi, um Produktionstechnologien zu charakterisieren, die im Nanometer-Bereich angesiedelt sind (Taniguchi 1974). Sein Aufsatz erschien in einer abgelegenen Konferenzdokumentation und blieb seinerzeit ohne Wirkung. Eric Drexler, Physiker, wichtiger Ideengeber der Nanotechnologie in einer breiten Öffentlichkeit und heute die umstrittenste Gestalt in der Geschichte der Nanotechnologie, konkretisierte in den 1980er Jahren die Vorstellung, die Dinge ,aus den kleinsten Teilchen“ aufzubauen durch das theoretische Konzept, Objekte Molekül für Molekül mittels einer Sequenz präzise gesteuerter chemischer Reaktionen zusammenzufügen. Um zu einer industriellen, massenhaften Produktion zu kommen, müssten diese Nanomaschinen (Assembler) in der Lage sein, Kopien ihrer selbst herzustellen, sich also selbst zu replizieren (Drexler 1987). In den 1980er Jahren wurde der Physik-Nobelpreis für die Entwicklung von Techno- 
logien zur Sichtbarmachung des Mikro- und Nanokosmos vergeben (an Ernst Ruska, Gerd Binnig und Heinrich Rohrer). Im Jahr 1989 gelang es im IBM Forschungszentrum Almaden in Kalifornien erstmals, Atome direkt zu positionieren (allerdings unter extremen und hoch spezifischen Bedingungen: Eigler 1999). Aus 35 Xenon-Atomen 'schrieben' die Wissenschaftler symbolträchtig den Namen IBM auf eine Oberfläche. Dieses Bild, das 1990 in der britischen Wissenschaftszeitschrift Nature (Eigler/Schweizer 1990) erschien, repräsentierte fortan einen Meilenstein in der Geschichte der Nanotechnologie. Nachdem Konzept, Begriff und Instrumente vorhanden waren, schien die Zeit nun reif, industrielle Anwendungen zu entwickeln (National Science and Technology Council 1999; BMBF 2004b).

Der geschilderten traditionellen Ideengeschichte lässt sich mit Foucault eine Untersuchung der Ereignisse, der Serien dieser Ereignisse, ihrer Regelhaftigkeit und ihrer Möglichkeitsbedingungen entgegensetzen (Foucault 1991: 35). Feynmans Idee lässt sich zwar als Ursprung erzählen, doch wurde die Idee erst zu diesem Ursprung durch die Regelhaftigkeit der Bezüge insbesondere technologiepolitischer Akteure gemacht. Diskurse sind um Akteure, Ereignisse und Institutionen gruppiert bzw. generieren und transformieren diese, und niemand „kann in die Ordnung des Diskurses eintreten, wenn er nicht gewissen Erfordernissen genügt, wenn er nicht von vornherein dazu qualifiziert ist" (Foucault 1991: 35). So bietet sich der Rekurs auf einen Nobelpreisträger als Geburtsdokument eines Technologiefeldes an. Zugleich wird damit der Beginn in den USA, dem technologisch führenden Nationalstaat in der zweiten Hälfte des zwanzigsten Jahrhunderts, lokalisiert. Und schließlich kommt der Autor aus der Physik, der seinerzeitigen Leitwissenschaft. Im Kontext der heute herrschenden globalen Machtverhältnisse in Politik, Wirtschaft und Wissenschaft ist es eher unwahrscheinlich, dass es eine Ereigniskette in der Ordnung des Diskurses geben könnte, in der durch permanente diskursive Wiederholung die Idee einer afrikanischen Ingenieurin oder eines chinesischen Chemikers als Ursprung einer heutigen Schlüsseltechnologie ausgemacht würde. K. Eric Drexler, der mit seinem radikalen Konzept der „molekularen Nanotechnologie“ zwar wichtigster Ideengeber des Nano-Diskurses ist, aber zugleich auch ein radikaler Außenseiter blieb, machte Feynmans Idee populär (Drexler 1987) und stellte sein befremdlich revolutionäres Konzept damit in eine unabweisbar durch den Nobelpreisträger legitimierte Traditionslinie.

Der Rekurs auf Feynman vervielfältigte sich im diskursiven Selektionsprozess, der Anfang der 1990er Jahre im technologiepolitischen Umfeld führender Industriestaaten durch Konferenzen und Studien zur technologischen Früherkennung (die eher Entwurf als Erkennung darstellt) in Gang gesetzt wurde. Im Laufe dieser Jahre wurde damit die Identität des Feldes formiert, das heute unter den Begriff der Nanotechnologie subsumiert wird. Was heute als Einheit der Nanotechnologie erscheint, besteht insofern aus einer Serie von diskursi- 
ven Ereignissen, deren gemeinsame Bezugspunkte durch Wiederholung und Intertextualität produziert werden. Das Ziel ist die Kontrolle von Materie auf der Nanoebene, also auf der Ebene von Molekülen und Atomen. ${ }^{5}$ Erst mit dem Beginn technologiepolitischer Aktivitäten führender Industriestaaten in den neunziger Jahren wird Nanotechnologie konzeptionell zu einem viel genutzten Begriff in verschiedenen Wissenschaften und in der breiten Öffentlichkeit. ${ }^{6}$ Die Fördermittel des BMBF stiegen vom Beginn einer dezidierten Nanoförderung im Jahr 1998 von 27,6 Mill. Euro auf 134,4 Mill. Euro im Jahr 2006 (Bundesregierung 2006: 2) und boten somit zunehmend Anreize für unterschiedliche wissenschaftliche Disziplinen wie für Industrieforschung, die eigenen Forschungen auf den technologiepolitischen Rahmen der Nanotechnologie zu beziehen. Die politische Ökonomie der Nanotechnologie lässt sich in diesem Sinne als eine politische Ökonomie des Versprechens ${ }^{7}$ charakterisieren, die nur funktionieren kann, wenn sich die Versprechen verbreiten und vervielfältigen, wenn sie allerorten aufgegriffen und konkretisiert werden.

\section{Stand und Status der Versprechen}

Die politische Aufmerksamkeit in Verbindung mit stark steigenden öffentlichen Forschungsmitteln transformiert die Versprechen in einen breiten wissenschaftlichen und öffentlichen Diskurs. Steigende öffentliche Fördermittel ziehen Interesse aus unterschiedlichen Branchen und Disziplinen nach sich, die Ausweitung und zugleich Konkretisierung einer potentiellen In-Wert-Setzung des Nanokosmos bestätigt und forciert den Fluss von Forschungsgeldern. In der Förderpolitik führender Industriestaaten lässt sich eine hohe zeitliche Übereinstimmung feststellen. Zwischen 1998-2002 wurden umfassende Programme etabliert, die nicht zuletzt aus einer permanenten wechselseitigen Evaluierung nationalstaatlicher Technologiepolitiken resultierten und damit so-

5 Die Definition der Nanotechnologie, die vom BMBF verwendet ist, ist entsprechend breit (wie auch Definitionen im internationalen Kontext): „Nanotechnologie beschreibt die Herstellung, Untersuchung und Anwendung von Strukturen, molekularen Materialien, inneren Grenz- und Oberflächen mit mindestens einer kritischen Dimension oder mit Fertigungstoleranzen (typischerweise) unterhalb 100 Nanometer. Entscheidend ist dabei, dass allein aus der Nanoskaligkeit der Systemkomponenten neue Funktionalitäten und Eigenschaften zur Verbesserung bestehender oder Entwicklung neuer Produkte und Anwendungsoptionen resultieren. Diese neuen Effekte und Möglichkeiten sind überwiegend im Verhältnis von Oberflächen- zu Volumenatomen und im quantenmechanischen Verhalten der Materiebausteine begründet“ (BMBF 2004a: 7).

6 Der Effekt zeigt sich insbesondere in einem parallelen Anstieg von Nano-Forschungsmitteln und wissenschaftlichen Nano-Publikationen: Die Datenbank Medline, in der wichtige medizinische und naturwissenschaftliche Publikationen indexiert sind, verzeichnet 1997 gerade einmal 15 Artikel zu Nanotechnologie, im Jahr 2000 sind es 105, 2003 bereits 1.870, also mehr als eine Vertausendfachung in sechs Jahren.

7 Auch in der französischen Diskussion wird von einer „économie des promesses“ in Bezug auf die Nanotechnologie gesprochen (Joly/Callon et al. 2005), allerdings wird dabei die „Ökonomie der Versprechen“ als Teil einer erneuten linearen Fortschrittskonzeption kritisiert. 
wohl Effekt von internationaler Konsultation als auch Konkurrenz sind. In den nanotechnologiepolitischen Strategiepapieren der nationalstaatlichen Initiativen werden kontinuierlich die Strategien anderer Staaten ausgewertet und die wachsenden Fördermittel gelten jeweils als zentrales Argument, das je eigene Programm auszuweiten. So setzt die Konkurrenz eine Spirale steigender Finanzmittel in Gang.

Banken greifen die Visionen auf, stellen fest, dass sich zwar keine quantitativen Prognosen zum Marktpotential wagen lassen, die wirtschaftlichen Chancen jedoch enorm seien, wenn sich die Versprechungen der molekularen Nanotechnologie auch nur halbwegs bewahrheiten würden. Nachdem seit 1998 durch öffentliche Fördermittel und die Ausschreibung von Nano-Kompetenzzentren Nanotechnologie als Leitbild etabliert wurde und eine "Nano-Szene“ entstanden ist, werden die folgenden - ebenfalls öffentlich geförderten Marktanalysen auf der Grundlage der Erwartungen eben dieser Akteure erstellt. Bereits auf dem Feld tätige Forschende aus Universitäten, Forschungseinrichtungen und Unternehmen, die mit den öffentlichen Fördergeldern forschen und entwickeln, werden hinsichtlich ihrer Markteinschätzung befragt. Somit werden die Erwartungen potenziert: „Das Weltmarktvolumen von Produkten, in denen nanotechnologische Herstellungsverfahren oder Komponenten einen wesentlichen Beitrag liefern, liegt bereits heute in der Größenordnung von etwa $100 \mathrm{Mrd}$. Euro. Und dies mit stark steigender Tendenz!" (Luther/Bachmann et al. 2006: 5). Bis zum Jahr 2015 sollen es bis zu 1 Billion Euro sein (BMBF 2006: 11). Allerdings gibt es in Deutschland bisher lediglich ungefähr 200 Start-Up Nano-Unternehmen, deren Gesamtbeschäftigtenzahl auf insgesamt 5.000 Mitarbeiter geschätzt wird - noch dazu verzeichnet die Hälfte der Start-Ups einen Jahresumsatz von weniger als 0,5 Mio. Euro Umsatz (Luther/Bachmann et al. 2006: 25). Die wichtigste Ressource der Technologiepolitik, auf die sie setzt und die sie zu vervielfältigen sucht, ist damit der Glaube an die Zukunft der Nanotechnologie, der aus mittelfristig am Markt zu erwartenden Produkten und Verfahren gespeist wird, die zugleich eine Brücke zu den visionären Anwendungen in fernerer Zukunft bilden.

Der Umgang mit mittel- und insbesondere langfristigen Zukunftsoptionen ist dabei in verschiedenen Branchen unterschiedlich ausgeprägt: Innerhalb der Elektronik/Halbleiterindustrie werden internationale Roadmaps für die zukünftige technologische Entwicklung entworfen, die sämtliche Aspekt der Chipherstellung umfassen. Nanotechnologie wird dabei entscheidend sein, um eine weitere und weit reichende Miniaturisierung zu gewährleisten (z.B. über nichtoptische Nanolithografie, aber auch alternative Ansätze). Die Chemieindustrie gilt als eine der Branchen, die sich am stärksten durch Nanotechnologie verändern wird, wobei die Ziele und Hauptanwendungen vielfältiger aber auch weniger absehbar sind. Im nanoskaligen Bereich verändern sich die Eigenschaften von Stoffen, so dass sich Schmelz- und Siedepunkt verschieben, die 
chemische Reaktivität steigt durch die extrem vergrößerte Oberfläche, womit zum Beispiel die katalytische Ausbeute steigt und neue Anwendungen möglich werden. Mit lange etablierten nanostrukturierten Materialien wie Carbon Black, Kieselsäure oder Polymerdispersionen werden bereits Milliardenumsätze am Weltmarkt erzielt, bei neueren Nanomaterialien wie Kohlenstoffnanoröhren, Polymernanokompositen, Aerogelen, organischen Halbleitern und anorganischen Nanopartikeln, deren Marktvolumen auf derzeit ca. 2 Mrd. Dollar geschätzt wird, wird eine hoch dynamische Entwicklung erwartet. Eng mit der Chemie verknüpft sind neue Möglichkeiten in der Optik wie schaltbare Spiegel oder hocheffiziente Dünnfilmsolarzellen. Unter Nanooptik wird die Ultrapräzisionsbearbeitung optischer Komponenten verstanden. Präzisionsoptiken kommen vor allem in der Lithografie zur Anwendung wo sie für die Herstellung elektronischer Bauteile mit immer geringeren Strukturbreiten unabdingbar sind. Das Marktvolumen von Nanotechnologien in der Optischen Industrie wird auf aktuell auf ca. 10 Mrd. Dollar geschätzt (Luther 2004; Luther/ Bachmann et al. 2006).

Diese heterogenen technischen Entwicklungen konzeptionell als Nanotechnologie zusammenzufassen, ist nicht primär naturwissenschaftlich begründet, sondern strategisch. Da die soziale, politische und ökonomische Reflexion neuer Technologien heute nicht mehr der technischen Entwicklung nachfolgt, sondern Teil der Entwicklung von Schlüsseltechnologien ist, bekommen Diskurse, die die zukünftige Entwicklung antizipierend beschreiben bzw. Szenarien entwerfen, einen neuen Status: Reflexion und Antizipation begleiten nicht nur die Technikentwicklung, sie formieren das Technologiefeld. Technikfolgenabschätzung und Innovationsstudien analysieren nicht retrospektiv die Auswirkungen vermeintlich feststehender Technologien, sondern sind konstitutiv für die Entwicklung neuer Technologien.

Die Amalganisierung von Wissenschaft, Gesellschaft, Politik und Ökonomie in der Entwicklung von Technologien, die traditionelle Grenzen überschreiten, wird in der (de)konstruktivistischen Technikforschung als „Technoscience“ gefasst. „Technoscience extravagantly exceeds the distinction between science and technology as well as those between nature and society, subjects and objects, and the natural and the artifactual that structured the imaginary time called modernity" (Haraway 1997: 5). Nanotechnologie ist ein zentrales Feld von Technoscience-Visionen, denn mit der Perspektive, Prozesse auf der Nanoebene der Atome und Moleküle zu beherrschen, wären nicht nur eine „neue industrielle Revolution“ möglich, sondern darüber hinaus die umfassende Kontrolle, Inwertsetzung und Verwertung organischer „natürlicher“ Prozesse (insbesondere in der Verbindung von Nanotechnologie, Biotechnologie etc.: Roco/Bainbridge 2002; kritisch: ETC Group 2003a). Was langfristig technisch möglich sein wird, welche Produkt- und Verfahrensinnovationen sich konkret ergeben könnten, ist kaum abzuschätzen, aber Gegenstand von 
TA-Studien und Regierungsprogrammen (vgl. z.B. TAB 2003; President's Council of Advisors on Science and Technology 2005). Ebenso ungewiss ist es, aus welcher transdisziplinären Zusammenarbeit zwischen Physik, Chemie, Biologie und Ingenieurwissenschaften und in welchen Branchen und Anwendungen diese zu hohem Wachstum führen werden. Aus dieser unberechenbaren Dynamik mit vielen Unbekannten ergibt sich für die staatliche Technologiepolitik, dass eine Steuerung, die Planbarkeit voraussetzt, nicht möglich ist. Staatliche Politik steht allerdings im Zentrum der Governance von Nanotechnologie, die sich im Zusammenspiel staatlicher und privater Akteure herausbildet und dabei jene Ein- und Ausschlüsse produziert, die das Feld der Nanotechnologie strukturieren und formieren.

Dabei sind die Governance-Formen, die sich in der politischen Ökonomie der Nanotechnologie herausbilden, um drei Ein- und Ausschließungssysteme (angelehnt an Foucault 1991) gruppiert, die im Folgenden dargestellt werden: Die Governance von Wahrheit besteht in der Organisation der Grenzen des Technologiefeldes, um den äußersten Rand des mittelfristig technisch Möglichen auszuloten und jene Bereiche als 'Science Fiction' zu klassifizieren, die als unmöglich gelten. Beim zweiten System handelt es sich um die Governance der Relevanz, in deren Rahmen die ökonomisch relevanten der technisch möglichen Optionen bestimmt werden. Drittens schließlich lässt sich eine Governance der Sicherheit feststellen, in der sowohl ökonomische Risiken als auch Umwelt- und Gesundheitsrisiken verhandelt werden.

\section{Die Governance von Wahrheit: Was ist Nanotechnologie?}

Wie weit die technologiepolitisch und industriell intendierte technische Beherrschung atomarer und molekularer Prozesse sowie die entsprechende Inwertsetzung des Nanokosmos mittelfristig möglich sein wird und wo die Grenze zum Science-Fiction-Genre beginnt, ist zwar keineswegs mit Sicherheit zu bestimmen, aber trotzdem Gegenstand zentraler, wenn auch mit vielen Unwägbarkeiten verbundener technologiepolitischer Langfrist-Programmatik. Die Governance der Nanotechnologie besteht nun erstens darin, die Grenzen und damit die Reichweite des Versprechens auszuhandeln und zu bestimmen: Was ist Nanotechnologie, wo endet die Nano-Wissenschaft, wo beginnen die Märchen und die unrealisierbaren Nano-Fiktionen? Als Governance der Wahrheit lässt sich dieser Prozess charakterisieren, da die Produktion des handlungsrelevanten Wissens nicht auf die notwendigerweise vage Bestimmung von wahrscheinlichen Zukünften gerichtet ist, sondern einen (wenn auch nur temporär gültigen) Wahrheitsanspruch erhebt, der auf die Legitimation von Ausschließung gerichtet ist. Wahrheit wird zusammen mit Machtpraxen 'produziert' und ist insofern eine Wahrheitspolitik im Sinne Foucaults.

Bei der diskursiven Konstituierung des Technologiefeldes legitimieren Politik 
und Wissenschaft die von ihnen temporär gezogenen Grenzen zwischen Science und Fiction sowie ihre Arbeitsteilung zur Entwicklung der Nanotechnologie wechselseitig: Ist schon Wissenschaft ein Feld, dass sicher durch die permanente Verhandlung seiner Grenzen zur Nicht-Wissenschaft erst konstituiert (Gieryn 1995; Gieryn 1999), so gilt dies auch für das breite wissenschaftliche und technologische Feld der Nanotechnologie: Die wissenschaftlichen Akteure, die in den Prozess der Grenzziehung eingebunden sind, profitieren erstens von ihrer „Grenzarbeit“, da ihre spezifischen Forschungsfelder Teil der Förderprogrammatik werden. Zweitens sind sie als Akteure in dem technologiepolitischen Netzwerk privilegiert und können mindestens von Vertrauen und Wohlwollen seitens des Geldgebers bei der Begutachtung von eigenen Forschungsvorhaben ausgehen. Und drittens steigt ihre Reputation in wissenschaftlichen Institutionen, die sich immer stärker über Drittmittel finanzieren müssen, sowie durch die politischen Netzwerke, die einen privilegierten Ressourcenzugang erwarten lassen. In dieser Governance der Wahrheit entstehen die ersten Umrisse einer politischen Ökonomie des Versprechens, indem Grenzziehungen zwischen wahrscheinlichen und realistischen Entwicklungen auf der einen Seite und unrealistischen Fiktionen auf der anderen Seite etabliert werden. Die zukünftigen wissenschaftlich-technischen Grenzen sind in der jeweiligen Gegenwart unentscheidbar, wie ein Blick in die Zukunftsszenarien aus der Wissenschaft der vergangenen Jahrzehnte zeigt - die übrigens keine Vorstellung einer Nanotechnologie enthalten (vgl. z.B. Helmer/Gordon 1967; Kahn/Wiener 1971). Wenn aber die politische Ökonomie des Versprechens gerade auf den Langfristoptionen beruht, so ist das Grenzgebiet zwischen Science und Fiction zentral für die Konstituierung des technologiepolitischen Feldes und seiner Grenzen. Die nanotechnologische „Neuschöpfung der Welt" („Shaping the World Atom by Atom“ National Science and Technology Council 1999), die als Vision von der US-amerikanischen Nanotechnologie-Initiative vertreten wird, mag weniger dem entsprechen, was Naturwissenschaftler erreichen zu können meinen, als vielleicht ein Mittel der Akzeptanzbeschaffung durch das Ausmalen einer ,schönen neuen Welt' sein (Nordmann 2003). Doch sie fungiert als mobilisierender Faktor. Mit den weit reichenden positiven Visionen ist allerdings immer auch die Kehrseite ihrer spiegelbildlichen Horrorszenarien verbunden (Coenen 2004; TAB 2004: 145ff). So gehört zur politischen Ökonomie des Versprechens zugleich die politiknahe Weiterentwicklung des Instrumentariums zur Bewertung von Versprechen und Visionen, die von anwendungsorientierten Forschungsinstituten und der wissenschaftlichen Politikberatung wahrgenommen wird, die die Technologiepolitik gleichermaßen mit gestalten. ${ }^{8}$ Wie es in der Forschung zu technischen Leitbil-

8 In der deutschen Nanotechnologiepolitik gehören zu diesen Institutionen insbesondere das Technologiezentrum des Vereins Deutscher Ingenieure (VDI-TZ), das die Nanotechnologiepoli- 
dern in den 90er Jahren eine „praxeologische“ Richtung gab, in der Leitbilder nicht nur als analytisches Instrument, sondern auch als praxeologisches und normatives Instrument genutzt wurden (vgl. Canzler/ Dierkes 2001: 461), so werden Visionen ebenfalls zu einem Instrument, Politiken zu fokussieren. Methodisch expliziert wird diese Herangehensweise aktuell im Vision Assessment, das im Kontext der staatlichen Technologiepolitik entwickelt wird (Grunwald 2004). Vision Assessment soll dazu dienen, „gleichsam die Spreu vom Weizen zu trennen", um sinnvolle und weniger sinnvolle Leitbilder voneinander zu unterscheiden (Coenen 2004: 84). Vision Assessment ist insofern eine weitere Ebene des stetigen Kommentars, durch den Diskurse erst zu ebensolchen werden (Foucault 1991: 16f), wobei die variantenreiche Wiederholung und Wiederkehr von Motiven die Redundanz erzeugt, die bestimmte Positionen dauerhaft einhegt, andere ausschließt und gleichzeitig durch stets modifiziert reproduzierte Positionen flexibel darauf reagieren könnte, dass sich so manche Prognose schnell als überholt herausstellt. Vision Assessment geht nicht über bestehende Methoden hinaus - wie über die aufwändige Delphi-Methode, die auf einem mehrstufigen, qualitativen Prognoseverfahren beruht, in dem Expertengruppen systematisch befragt werden (zur Methode s. Grunwald 2004). Insofern wird hier nur ein weiterer Gegenstand in den Wahrheitsdiskurs integriert. Radikale technische Visionen entstehen primär außerhalb der etablierten technologiepolitischen Diskurse und verdanken ihre Ausstrahlungskraft gerade ihrem überschießenden Gehalt an Neuem (z.B. Drexlers Buch Engines of Creation: Drexler 1987). Nun werden sie im Kontext einer politischen Ökonomie des Versprechens jedoch Prozeduren unterworfen, die darauf ausgerichtet sind, ihre Kräfte zu bändigen, die Zufälle ihres Auftauchens zu beherrschen und sie auf die Form der gegenwärtigen technologiepolitischen Rationalität zu begrenzen. Die Wahrheitsproduktion in der Nanotechnologiepolitik ist dabei asymmetrisch auf die positiven Versprechen reduziert und schließt die negativen - die sich logisch und technisch immanent aus den positiven Szenarien ergeben - weitgehend aus.

\section{Die Governance der Relevanz: Was sind die zukünftigen Märkte der Nanotechnologie?}

Wissenschaftlich-technische Machbarkeit ist allerdings nur der erste Mechanismus für Einschluss und Ausschluss auf dem technologiepolitischen Feld. Als zweite Grenzlinie fungiert die Herstellung von Relevanz. Nicht alles, was als wissenschaftlich ertragreich und technisch machbar klassifiziert wird, kann seine Relevanz und damit seine Förderfähigkeit unter Beweis stellen. Maßgeb-

tik des BMBF vorbereitet, unterstützt und organisiert, das Institut für Technikfolgenabschätzung und Systemanalyse (ITAS) am Forschungszentrum Karlsruhe[0] und das am ITAS organisatorisch angesiedelte Büro für Technikfolgenabschätzung am Deutschen Bundestag (TAB). 
liches Relevanzkriterium ist in der heutigen Technologiepolitik das Kriterium des zukünftigen Markterfolges und das prognostizierte Marktvolumen (BMBF 2002b). Der institutionelle Rahmen, um der Frage nach den Märkten der Zukunft nachzugehen, wird durch das Technology Assessment (TA) gebildet: Der deutsche Begriff der Technikfolgenabschätzung ist dabei umstritten, da er als wirtschaftsfern gilt und mit fehlender Technikakzeptanz in Verbindung gebracht wird. Stattdessen wird zunehmend (in der deutschen Politik durch den Geldgeber BMBF forciert) von Innovations- und Technikanalysen (ITA) gesprochen. Der Schwerpunkt der Technikfolgenabschätzung liegt auf der Früherkennung der wirtschaftlichen Möglichkeiten und nicht (mehr) auf der (womöglich kritischen) Abschätzung gesellschaftlicher Folgewirkungen - wobei die „Früherkennung" keine Erkennung gegebener Möglichkeiten darstellt, sondern ihrerseits wiederum eine Methode darstellt, gewünschte Zukunft durch Beschreibung spezifischer Möglichkeiten und gangbar erscheinenden Wegen in die anvisierte Zukunft zu konkretisieren und zu verbreiten. Technikfolgenabschätzung lässt sich auf dem Feld der Nanotechnologie mit unterschiedlicher Reichweite feststellen: Von den wirtschaftlichen Möglichkeiten und technischen Auswirkungen einzelner Nanotechnologien ${ }^{9}$ bis zu umfassenden Analysen, die technologie- und branchenübergreifend sowohl kurz- als auch langfristige Entwicklungsoptionen beleuchten. Technikfolgenabschätzung und staatliche Technologiepolitik sind eng verbunden, ${ }^{10}$ wobei technologiepolitische Akteure und Beobachter vielfach zusammenfallen, so dass die Reflexion wiederum stark auf das primäre Ziel der Erhöhung der Wettbewerbsfähigkeit gerichtet ist.

Regierungen oder Parlamente haben umfassende TA-Studien veranlasst, die sich nicht auf die Analyse von Technologien beschränken, sondern TechnikVisionen und konkurrierende gesellschaftliche Ansprüche an zukünftige Technologien untersuchen und bewerten (TAB 2003; Royal Society/The Royal Academy of Engineering 2004). Insofern sind TA-Studien keine „angewandte Wissenschaft“ in einem objektivistischen Verständnis, sondern (machtvolle) argumentative Beiträge zur weiteren Entwicklung der Governance von Nanotechnologien. TA erbringt in der Form als Policy-Analyse Interpretations- und Übersetzungsleistungen zwischen unterschiedlichen Disziplinen, allgemeinen Problembeschreibungen und wissenschaftlichen Fragestellungen sowie zwischen Wissenschaft, Öffentlichkeit und Politik (Saretzki 2005: 464).

9 In Deutschland werden diese Studien insbesondere vom VDI-TZ in Düsseldorf erarbeitet. Zu den neueren Studien gehören z.B. (Eickenbusch/Hoffknecht et al. 2003; Wagner/Wechsler 2004).

10 So führt das VDI-Technologiezentrum in Düsseldorf im Auftrag des BMBF zahlreiche Studien zu Technikfolgenabschätzung durch, fungiert als Projektträger für das BMBF und bedient sowohl technische Fachöffentlichkeiten als auch die allgemeine Öffentlichkeit mit zielgruppenspezifischen Informationen. Neben den technischen Berichten (z.B. Bachmann 1994; 1998; Luther 2004; Luther et al. 2004) sind auch die Informationsbroschüren des BMBF vom VDI-TZ und die Materialien des „Nanotruck“ (BMBF 2004b; VDI Technologiezentrum o. J.-a; VDI Technologiezentrum o. J.-b; VDI Technologiezentrum o. J.-c) vom VDI-TZ erstellt worden. 
Der Argumentationsrahmen der primär staatlich finanzierten TA ist normativ zumeist auf das prioritäre Ziel der beschleunigten Technikentwicklung und -diffusion begrenzt. ${ }^{11}$ Nanotechnologie soll der deutschen Wirtschaft Wettbewerbsvorteile im internationalen Wettbewerb bescheren und umgekehrt hänge die zukünftige Wettbewerbsfähigkeit Deutschlands von der Erschließung des Nanokosmos ab (BMBF 2002a; BMBF 2002b; BMBF 2005). In der Entwicklung einer politischen Ökonomie der Nanotechnologie ist TA der Modus, den politischen Handlungsraum abzustecken und die Konzentration öffentlicher Mittel auf spezifische Branchen und technische Schwerpunkte zu begründen und zu legitimieren. Wenn das zentrale politische Relevanz-Kriterium für die Entwicklung von Zukunftstechnologien darin besteht, welchen Beitrag eine Technologie für Wirtschaftswachstum und Wettbewerbsfähigkeit leistet, dann wird der potentielle Beitrag einer Technologie zur Lösung gesellschaftlicher Probleme, die sich nicht über den Markt organisieren lassen, nachrangig. In einigen Bereichen überschneiden sich gesellschaftliche Relevanz, erwarteter Profit und sogar positive Nachhaltigkeitswirkungen. Dies könnte der Fall bei der Optimierung von Solartechnik durch Nanotechnologie sein. Allerdings werden erhebliche öffentliche Mittel für Forschungsziele aufgewendet werden, deren gesellschaftliche Relevanz zweifelhaft ist, die aber (für den deutschen Standort) besonders wichtige Märkte adressieren, so zum Beispiel für „schaltbare“ Autolacke mit Farbwechsel (BMBF 2004a: 11).

Entgegen der programmatischen Nachhaltigkeits-Rhetorik zementiert die Nanotechnologiepolitik globale Ungleichheit: Mit der Patentpolitik und der Erweiterung von Patentierungsmöglichkeiten sollen eventuelle Vorsprünge gesichert werden (BMBF 2004a; Europäische Kommission 2004). Wird Nanotechnologie zur Schlüsseltechnologie, so hängen Produkt- und Verfahrensinnovationen direkt von ihr ab; die Möglichkeiten peripherer Staaten, an die technologische Entwicklung anzuschließen, verringern sich drastisch. Da Nanomaterialien bisherige Rohstoffe substituieren sollen (z. B. Gummi, Baumwolle) würde globale Ungleichheit verschärft (vgl. ETC Group 2004b).

Während das Wahrheitskriterium dazu dient, die Grenzen von Wissenschaft und technischer Machbarkeit zu bestimmen, dient das Relevanzkriterium da$\mathrm{zu}$, von allen unter Umstanden möglichen wissenschaftlichen und technischen Möglichkeiten diejenigen auszuwählen, die den wirtschaftspolitischen Kriteri-

11 Die einzige staatlich in Auftrag gegebene TA-Studie, die diese Ausrichtung durchbricht und ergebnisoffene partizipative Prozesse zur weiteren Technologiepolitik fordert, ist die britische Studie der Royal Society (Royal Society/The Royal Academy of Engineering 2004), deren Vorschläge dann auch Papier blieben (Royal Society 2005; UK Government 2005). Die Vorschläge der Royal Society beziehen sich sowohl auf konkrete inhaltliche Punkte als auch Verfahrensweisen. Gefordert wird Transparenz durch Lebenszyklus-Analysen von NanoStoffen, Behandlung von Nanopartikeln als neue Substanzen im Kontext der europäischen Chemikalien-Gesetzgebung REACH mit entsprechenden Nachweispflichten, hohe Sicherheitsüberprüfungen für Verbraucherprodukte sowie Produktkennzeichnung. 
en und Machtverhältnissen entsprechen. Diese Norm erscheint allerdings als Nicht-Norm - als schiere Notwendigkeit. Zwar wird den Nanowissenschaften und -technologien gern ein „revolutionäres Potential“ zugeschrieben, doch soll eben dieses genutzt werden, um die Sackgasse von stetigem Wachstum doch endlos verlängern zu können.

Hier zeigt sich ein eklatanter Widerspruch innerhalb der politischen Ökonomie des Versprechens: Das Versprechen der Nanotechnologie besteht darin, das Problem der Ressourcenknappheit vollständig zu überwinden. Für den radikalen Nano-Visionär Drexler ist es ein zukünftiger ,grüner Reichtum, der sich mit dem Reichtum der Natur vereinbaren lässt" (Drexler/Peterson et al. 1991: 193f); das BMBF verspricht eine solarbasierte dezentrale, umweltschonende Energieversorgung (vgl. BMBF 2002a: 13) und selbst Greenpeace berichtet von neuartigen Materialien, die ohne Umweltverschmutzung hergestellt werden können (Greenpeace Magazin 2000). Radikal bei Drexler und moderat beim BMBF (BMBF 1998) werden Zukunftswelten dargestellt, in denen die hochkomfortable Lebensweise des Industriezeitalters im Einklang mit einer (idealisierten) Natur steht; bei Drexler sogar für die gesamte Weltbevölkerung. In einer weltpolitischen Situation zugespitzter Ressourcenkonflikte wird die Nanotechnologie so zum Versprechen der Lösung von Ressourcenproblemen, obwohl diese durch die aktuelle Technologiepolitik doppelt verschärft werden: Erstens durch neue Ressourcenkonflikte, z.B. durch Substitution von Rohstoffen, die Patentierung (und damit Exklusivität von Verwertung) und die Machtkonzentration, falls die hochfliegenden Pläne von Unternehmen realisiert werden (vgl. ETC Group 2004b; ETC Group 2005), die voraussichtlich globale Ungleichheit verschärfen. Wenn es zweitens gelingen sollte, die technologiepolitische Strategie „Stärken zu stärken“, umzusetzen, also Exportüberschüsse weiter zu steigern und (insbesondere in der deutschen Politik) die Autoindustrie zu stärken, wird die Pfadabhängigkeit der bisherigen industriellen Entwicklung verstetigt.

\section{Die Governance der Sicherheit: Regulierung als Entwicklung von Nano-Märkten}

Die Governance von 'Sicherheit' schließlich bildet das dritte System zur Konstituierung einer politischen Ökonomie der Nanotechnologie. Dass Sicherheit politisch hergestellt werden soll, ist unumstritten, wie dies geschehen soll hingegen nicht. Nanotechnologie ist in der breiten Öffentlichkeit noch wenig bekannt (European Commission 2005b: 13 ff; Gaskell/Eyck et al. 2005), wird weitgehend positiv wahrgenommen ${ }^{12}$ und zeichnet sich bisher durch die Abwesenheit von polarisierten Konflikten aus (Schaper-Rinkel 2006a).

12 Nanotechnologie ist zwar noch viel unbekannter als z.B. Biotechnologie (European Commission 2005b), doch nimmt das Wissen (nicht zuletzt durch die Öffentlichkeitsarbeit der staatlichen Technologiepolitik) schnell zu. Ein Zuwachs an Wissen bedeutet dabei nicht un- 
Da technische Entwicklung heute in einer Widersprüchlichkeit von Chancen und Risiken verortet wird, sollen geeignete Governance-Formen die widersprüchlichen Anforderungen von Beschleunigung und Ausweitung der technischen Entwicklung einerseits und Vermeidung von Risiken andererseits gewährleisten (vgl. Grande 2001). Zwei Ziele werden in der Governance von Sicherheit politisch bearbeitet: Erstens 'Sicherheit vor gefährlichen Nanopartikeln' und zweitens die Sicherheit, dass (lax formuliert) „Nano drin ist, wo Nano drauf steht“. Wenn Nanotechnologien in Form neuer Produkte und Verfahren produziert und gehandelt werden sollen, so bedarf es dazu dieser zwei verschiedenen Formen von Sicherheit, die eng miteinander verknüpft sind: Erstens wird über Sicherheit in der Produktion und Anwendung von Nanotechnologien verhandelt und dabei festgelegt, ob mit der bisherigen Regulierung der Arbeits-, Gesundheits- und Umweltschutz gewährleistet ist bzw. wo Regulierungslücken festzustellen sind und wie diese gegebenenfalls zu schließen sind.

Was hier als Sicherheit politisch verhandelt wird, besteht darin, Spielregeln für den Umgang mit Risiken festzulegen und Regeln zu verhandeln, wie Risiken erhoben, klassifiziert und bearbeitet werden sollen (vgl. Schaper-Rinkel 2006b). Zweitens geht es um die Sicherheit bei der Qualität spezifischer Nanotechnologien (zurzeit im Hinblick auf Nanopartikel und -materialien). Dabei besteht die Aufgabe darin, Terminologien, Klassifizierungen und Standards für spezifische Nanotechnologien zu entwickeln, um die Herausbildung von industriellen Märkten für Nanoprodukte zu forcieren. Durch und mit den Definitionen, Abgrenzungen und Terminologien werden Eigentumsansprüche an Technologien über Patente möglich, so dass diese Prozesse eine Grundlage für die Inwertsetzung von Nanotechnologien bilden. Die Patentierung und Inwertsetzung von Nanotechnologien, insbesondere Nanopartikeln, hat allerdings auch für die an der Verwertung interessierten Unternehmen eine Kehrseite: Wenn hochspezifische patentierte Nanopartikel in der Zukunft zu Gesundheits- oder Umweltschäden führen, wären sie entsprechend präzise zurückzuverfolgen, was für Rückversicherungen ein erhebliches Risiko darstellt, weshalb Versicherungen die Risikodiskussion der Nanotechnologie (anders als die staatliche Forschungsförderung) vorangetrieben haben: „Es ist zu befürchten, dass die $\mathrm{Na}$ notechnologie zur Kategorie der revolutionären Risiken mit ursächlich nachweisbarer Schadenfolge gehören wird. Dabei sind die potenziellen Schäden in Bezug auf ihre Grösse und Raum/Zeit vermutlich nicht oder nur äusserst schwer abschätzbar" (Swiss Re 2004a: 40).

Der Bedarf, 'Sicherheit' politisch herzustellen, stellt sich erst dann heraus,

bedingt eine positive Einstellung gegenüber der Technologie (Lee et al. 2005), doch insgesamt lässt sich eine hohe Aufgeschlossenheit der Öffentlichkeit in bisherigen Studien feststellen (Gaskell et al. 2005). Studien zu Nanotechnologie-Berichterstattung in Publikumsmedien zeigen, dass die Berichterstattung sowohl in den USA, Großbritannien wie auch die deutschsprachige Berichterstattung in Publikumsmedien positiv sind (Grobe et al. 2005; Stephens 2005). 
wenn überhaupt Risiken thematisiert werden. Während die Thematisierung von Risiken in der sozialwissenschaftlichen Technikforschung, der Risikoforschung und auch in der Technikfolgenabschätzung als unumgänglich und sinnvoll im gesellschaftlichen Umgang mit Technik gilt (EEA 2001; Marcinkowski 2001; Saretzki 2001), gilt das Thema Risiken in der deutschen (BMBF 2004a) und in der europäischen Politik als Gefahr für die erfolgreiche Durchsetzung der Nanotechnologie (Europäische Kommission 2004). In den ersten Jahren der deutschen Nanotechnologiepolitik (1998-2003) wurden Risiken und Negativszenarien sowohl von Parteien, Regierung und dem Umfeld der parlamentarischen TA als Gefahr für die Akzeptanz der Technologie gesehen und tabuisiert. Doch bereits die Geschichte der Gentechnik zeigt (Gottweis 1998; Gottweis 2005), dass die Risikodiskussion letztlich (ohne dass dies der Intention der Gegner entsprach) produktiv und formierend wirkte, und nicht etwa bremsend, wie in der offiziellen Regierungspolitik und dem common sense der Wirtschaftsberichterstattung nach wie vor gemutmaßt wird. Auch in der Nanotechnologiepolitik zeigt sich bisher, dass die Risikodiskussion hochproduktiv für die Herausbildung einer Governance der Nanotechnologie ist. Risiken erzeugen einen Handlungsdruck, den die leuchtenden Zukunftsszenarien aus den Broschüren der Forschungsprogramme nicht erzeugen, insbesondere in den Bereichen, in denen die technologische Entwicklung bereits weit fortgeschritten ist (aktuell: Nanopartikel). Schließlich könnte ein einziger Fall von Nanopartikeln, die sich als gefährlich erweisen und negative gesundheitliche Auswirkungen haben, die gesamte Technologie in Frage stellen und die Markteinführung von Nanotechnologie-Produkten insgesamt stark hemmen.

Um überhaupt Regulierungsnotwendigkeiten feststellen zu können, ist eine Spezifizierung von konkreten Nanotechnologien unabdingbar. ${ }^{13}$ Somit ist die Entwicklung einer Nomenklatur zentral für die Governance von Sicherheit. Mittlerweile gilt als common sense, dass die bisherige Regulierung insbesondere im Hinblick auf Umwelt-, Gesundheits- und Arbeitsschutz überprüft werden muss, da Nanopartikel aufgrund ihrer geringen Partikelgröße besondere Gefahrenpotentiale aufweisen und gängige Schutzvorkehrungen (Filter) nicht wirksam sind (Fraunhofer Institut für Silikatforschung/Innovationsforschung

13 In den Jahren 2004 bis 2005 erschienen wichtige Berichte und Dokumente zu Regulierungsanforderungen: Die Versicherungswirtschaft stellt Regulierungsanforderungen (Swiss Re 2004b; Allianz/OECD 2005), auf europäischer Ebene wird der Stand der Dinge zusammengetragen (Nanoforum Consortium 2004) und von einem breiten Spektrum von Akteuren aus international ausgerichteten Institutionen (International Risk Governance Council 2004) werden ebenfalls Regulierungsnotwendigkeiten formuliert. Zusammenfassende Darstellungen finden sich in: (Dürrenberger/Höck et al. 2004; Luther 2004). Während die meisten Ansätze auf eine Lösung durch Technik optieren und damit (was die generelle Ausrichtung anbelangt) unumstritten sind, ragt der Bericht der britischen Royal Society und Royal Academy of Engineering heraus, der eine umfassende gesellschaftliche Beteiligung (stakeholder) an der weiteren Technologieentwicklung fordert (Royal Society/The Royal Academy of Engineering 2004). 
2003; TAB 2003; Steinfeldt/Gleich et al. 2004: 182). Der Mangel an Daten und Wissen wird von allen Beteiligten als Problem angesehen, sowohl von NGOs, wie auch von Industrie, Behörden und den Rückversicherern. Auf dem Feld der Nomenklaturentwicklung treffen sich die Interessen der unterschiedlichsten Beteiligten. ${ }^{14}$ Die Klassifizierung von spezifischen Nanotechnologien (präzise Begriffsbestimmungen und Abgrenzungen) ist die Voraussetzung für Qualitätsstandards, somit für die industrielle Anwendung, für ein Nano-RisikoAssessment und -Management und für die Verhandlung von Regulierungsansprüchen. Eine Forderung, die trotz unterschiedlicher Interessen ungleiche Gruppen (von der Industrie bis zu radikalen NGOs, von Rückversicherern bis zu Umweltschützern) vereint, lautet somit, dass dieser Prozess durch öffentliche Förderung unterstützt werden soll: durch Förderung von konkreten Forschungsvorhaben, aber auch mittels Moderation des Prozesses durch spezialisierte Bundesbehörden oder Prozesse der regulierten Selbstregulierung wie bei der Normung.

Umstritten ist dagegen die Frage, wer mit welchen Ressourcen die entsprechenden Wissensbestände generiert und zur Verfügung stellt, die für eine Governance der Sicherheit notwendig sind. In der deutschen Politik wird weitgehend auf Freiwilligkeit gesetzt. Das Deutsche Institut für Normung (DIN) versucht, industrielle Akteure für die Arbeit in internationalen Standardisierungsgremien zu gewinnen. Ein zentrales Argument ist dabei der Wettbewerbsvorteil, den Unternehmen haben, wenn ihre Unternehmensstandards (die unternehmensweiten Regeln zur Qualitätssicherung in der Herstellung von NanoProdukten) zu internationalen Normen werden. Das Negativszenario lautet: „Wollen Sie in wenigen Jahren nach Normen produzieren, die aus China kommen?" Ein zweites Argument ist der Schutz von originären NanoProdukten gegenüber Produkten, die diesen Namen zwar führen, die aber gar nicht auf entsprechenden Innovationen beruhen (z.B. Standards für so genannte 'easy to clean'-Oberflächen). Hier zeigt sich ein Dilemma der erfolgreichen Programmatik des Versprechens, führte diese doch zu der staatlich anvisierten Aufmerksamkeit und sogar zu einem Nano-Hype, die wiederum eine Vielzahl von Trittbrettfahrern in den Marketingabteilungen aufspringen lässt: Nano ist zu einer Chiffre für Zukunft und Trend geworden, so dass mp3Player, Golfschläger, Nahrungsergänzungsmittel, Putzmittel und Kosmetik mit dem Label Nano beworben werden. Manche tragen nur den Namen, andere

14 Seit 2004 wurden entsprechende Initiativen in Gang gebracht, in Europa vgl. European Commission (2004a: 23); Malsch/Oud (2004: 77, 90); European Commission (2005a: 11); in den USA innerhalb des aktualisierten nationalstaatlichen Förderprogramms, vgl. National Science and Technology Council (2004); President's Council of Advisors on Science and Technology (2005: 45). In der Technikfolgenabschätzung (Luther 2004: 94 f) und in der Versicherungswirtschaft (Swiss Re 2004b: 37) wird die Entwicklung von Standards gefordert und auch die europäische Technologiepolitik nimmt sich der Entwicklung an (European Commission 2004b). 
versprechen mit dem Label Nano sensationelle Wirkungen, die sie nicht halten können und stellen für die politische Ökonomie des Versprechens eine doppelte Gefahr dar: Sollten Produkte, die mit dem Nano-Label werben ${ }^{15}$, enttäuschen, so könnte der Hype schnell in Enttäuschung umschlagen.

Damit steht die Frage nach einer möglichen Deklarationspflicht im politischen Raum, die - aufgrund der Auseinandersetzung um die grüne Gentechnik - erst als Schreckgespenst erschien (vgl. Schaper-Rinkel 2006a). Mittlerweile zeigt sich eine ambivalente Dynamik: Die Umweltgruppe THONG protestierte im Mai 2005 in Chicago vor der US-amerikanischen Bekleidungskette Eddie Bauer gegen Kleidung mit Nanotex-Fasern. Auf nackten Rücken war in Anlehnung an die Auseinandersetzung um Kernkraft und Gentechnik zu lesen: „Eddie Bauer - Hazard“ und „Expose the truth about NanoTech“. 2006 gab es in Deutschland (mit internationale Beachtung) zwei gegenteilige Skandale: Die Produkte des Unternehmens Neosino, das teure Nano-Produkte vertreibt, die vom Deutschen Sportbund empfohlen und vom FC Bayern München beworben wurden, enthalten laut einem Bericht des Fernsehmagazins Panorama keine Nanomineralien - der „Staub vom Bolzplatz“ habe vielmehr die gleiche Wirkung. Ende März 2006 wurden 97 zum Teil schwerwiegende Vergiftungsfälle nach der Anwendung eines „Nano“-Versiegelungssprays namens Nano-Magic gemeldet. Auch bei diesem Produkt blieb jedoch unklar, ob es überhaupt Nanopartikel enthielt. Mit diesen Fällen könnte die Kennzeichnung zu einem Mittel der Qualitätssicherung werden - statt zu einem Mittel, den Konsum vermeiden zu können.

Nanotechnologie soll nach der gemeinsamen Rhetorik unterschiedlicher Akteure „verantwortungsvoll“ entwickelt werden (um eine hohe Akzeptanz und damit schnelle Verbreitung zu gewährleisten); uneinig sind sich die Akteure hinsichtlich der staatlichen Handlungsnotwendigkeit und der Verbindlichkeit der Regeln (freiwillige Selbstverpflichtung versus Gesetze und Verordnungen). Grob lassen sich drei Positionen mit entsprechend divergierenden strategischen Schlussfolgerungen feststellen. Die erste - marktradikale - Position verbindet ihre positiven Zukunftserwartungen an die Nanotechnologie mit einem Glauben an die Selbstregulierung von Märkten. Sowohl Nano-Visionäre wie K. Eric Drexler (Drexler 1987) als auch deutsche und US-amerikanische Regierungsprogramme schreiben Nanotechnologien per se positive Wirkungen hinsichtlich Umwelt und Wirtschaft zu. Nanotechnologien ermöglichen demzufolge extreme Einsparungen bei Gewicht, Volumen, Rohstoff- und Energieverbrauch und würden so zu umweltschonender Produktion und Lebensweise beitragen (Drexler/Peterson et al. 1991; BMBF 2002a; BMBF 2004a: 27). Die

15 Was zum Beispiel überhaupt Nanopartikel genannt werden soll, ob es sich dabei fortan nur um synthetische Partikel handeln soll, oder ob auch 'natürliche' Nanopartikel (die z.B. in Autoabgasen enthalten sind, seit langem ein Forschungsgegenstand von Toxikologen) ist auch in Fachkreisen von Toxikologen höchst umstritten (vgl. Kurath/Maasen 2006). 
Gegenposition sieht in der Nanotechnologie ein hohes und unkalkulierbares Gefahrenpotential: Aktuell hinsichtlich der toxischen Wirkung von Nanopartikeln und -materialien und langfristig in Bezug auf Selbstorganisierung und -replikation von Nanostrukturen. Für diese Position steht insbesondere die kanadische Umwelt-NGO ETC-Group, die als politische Konsequenz ein Moratorium für alle Anwendungen der Nanotechnologie forderte und sich für Gremien einsetzt, die auf internationaler Ebene die Technologieentwicklung beobachten und bewerten (ETC Group 2002; 2003a; 2003c; 2004a; 2006). Drittens artikuliert sich schließlich eine auf Vorsorge orientierte Position, die zwar kein Moratorium fordert, aber verbindliche institutionelle Prozesse einfordert, über die Risikopotentiale überprüft und damit sichtbar werden. Diese Position wird im britischen Bericht zur Technikfolgenabschätzung vom Juli 2004 vertreten (Royal Society/The Royal Academy of Engineering 2004).

Konzeptionell wird in Europa auf das Vorsorgeprinzip (precautionary principle) als Grundlage für eine Governance der Sicherheit in der Nanotechnologie zurückgegriffen, während sich in den USA (zunehmend auch international) der Begriff der „Responsible Development of Nanotechnology“ (National Science and Technology Council 2004; vgl. Roco 2005) durchsetzt. In der USamerikanischen Nano-Förderinitiative werden darunter alle Aktivitäten gefasst, die sich mit Nachhaltigkeit, Umweltschutz sowie den 'gesellschaftlichen Aspekten von Nanotechnologie' beschäftigen (Roco 2004: 7f). Die weit verbreitete Rede von den gesellschaftlichen Aspekten der Nanotechnologie oder auch anderer Schlüsseltechnologien impliziert dabei eine Trennung zwischen gesellschaftlichen und nicht-gesellschaftlichen Bereichen der Technikentwicklung, was letztlich irreführend ist, da damit davon ausgegangen wird, es gäbe gesellschaftsfreie Aspekte der Technikentwicklung. Die Europäische Kommission nutzt ihn als Oberbegriff, in dessen Rahmen das Vorsorgeprinzip wirksam werden soll (European Commission 2004a: 20). Für die am stärksten mit Nano-Kritik international im Rampenlicht stehende kanadische NGO ETCGroup steht das Motto „Responsible Developments in Technologies“ für eine umfassende Kontrolle von Technologien im Kontext einer partizipativbasisdemokratischen Beteiligung aller gesellschaftlichen Gruppen (ETC Group 2003b: 10). Der kritische britische Bericht der Royal Society verknüpft mit dem Begriff ebenfalls ein hohes Maß an gesellschaftlicher Bestimmung technologischer Entwicklungen (Royal Society/The Royal Academy of Engineering 2004). Dass von den unterschiedlichen Akteuren ein gemeinsamer Begriff verwendet wird, zeigt die hohe Integrationswirkung der politischen Ökonomie des Versprechens. Dass darüber wirksame Mechanismen für Umwelt-, Gesundheits- und Verbraucherschutz entwickelt werden, ist bisher zu bezweifeln: Zwar werden zunehmend Dialoge über Risiken der Nanotechnologie geführt, doch erwachsen daraus bisher keine verbindlichen Regeln. Zum zweiten werden die Konzepte, die Verfahrensvorschläge für einen verbindlichen und fort- 
laufenden Regulierungsprozess machen, zwar umfassend gelobt, bleiben jedoch folgenlos (vgl. den Konflikt in Großbritannien: Royal Society/The Royal Academy of Engineering 2004; Royal Society 2005; UK Government 2005). Drittens schließlich ist die Governance der Nano-Sicherheit von einer defensiven Herangehensweise geprägt, indem es fast ausschließlich um die Vermeidung weiterer negativer Umweltwirkungen geht, während die aktive Förderung einer nachweislich ökologisch nachhaltigen Nanotechnologie schon deshalb marginalisiert ist, weil für eine solche keine Kriterien entwickelt werden. Insofern öffnet sich zunehmend eine Schere zwischen der rasanten Technologieentwicklung auf der einen Seite (resultierend aus den 4 Mrd. Euro öffentlicher Mittel und entsprechenden privaten Investitionen) und den langsam und nur begrenzt folgenreichen verlaufenden Diskursen auf der anderen Seite.

\section{Die Governance der Nanotechnologie: Konturen einer politischen Ökonomie des Versprechens}

So arbeitet die politische Ökonomie des Versprechens mit einer intendierten Self-fulfilling Prophecy. Während die wissenschaftlich-technischen Großprojekte des 20. Jahrhunderts - Raumfahrt und Atomkraft - noch mittels hierarchischer Steuerung von Oben und mit Hilfe der staatlichen Finanzierung gigantischer Forschungseinrichtungen realisiert worden sind, nutzt heute staatliche Technologiepolitik das gesamte Arsenal neuer Formen des Regierens, die gemeinhin unter den Begriff der Governance gefasst werden. Damit ändert sich der Status des Politischen in der Technologieentwicklung: Reflexive Diskurse wie Technikfolgenabschätzung, Technologiefrüherkennung, ethische Debatten und Technikfolgenabschätzung populärwissenschaftliche Darstellungen sind keine Begleitmaßnahmen für eine bereits feststehende Technologie, sondern formieren erst das Feld der Nanotechnologie.

Ist die Informationstechnologie aus Big Science entstanden, ganz im Geiste von Fordismus, nationalstaatlicher Planung und mit dem Zweiten Weltkrieg als einer entscheidenden Treibkraft, so entsteht die aktuell forcierte Schlüsseltechnologie des 21. Jahrhunderts unter den Bedingungen von Globalisierung, neuen Formen der Wissensgenerierung und einem von Regierungen vorangetriebenem nationalstaatlichen Wettlauf um Produkt- und Verfahrensinnovationen auf globalen Märkten, deren konkrete Form noch unbekannt ist. Forschung, Entwicklung und Kommerzialisierung der Nanotechnologie verlaufen immer weniger innerhalb nationalstaatlichen Grenzen, sondern sind global verteilt, stehen aber trotzdem im Fokus nationalstaatlicher Förderpolitiken, die damit den Standortwettbewerb forcieren. Zudem entsteht die Dynamik nanotechnologischer Innovationen in einem engen Wechselverhältnis von neuen Erkenntnissen der Grundlagenforschung, der Entwicklung neuer Instrumente und dem Wissenszuwachs höchst verschiedener Disziplinen, kurz in 
einem global verteilten und zugleich heterogenen Umfeld. Auch wenn es zentrale 'traditionelle' Akteure gibt, wie die Chemieindustrie (Nanomaterialien) oder die Chip-Industrie (Nanoelektronik), so kennzeichnet es die Nanotechnologie, dass das politisch angestrebte Innovationspotential sich nur dann realisieren lassen wird, wenn Technologien und Forschungsergebnisse aus verschiedenen Branchen und wissenschaftlichen Disziplinen zusammengeführt werden. Staatliche Technologiepolitik ist daher darauf ausgerichtet, heterogene Akteure anzusprechen und zusammenzubringen, um Innovationspotentiale sichtbar zu machen und um die Entwicklung von Produkt- und Verfahrensinnovationen zu beschleunigen. Die Beherrschung der 'Bausteine' des Lebens und der physikalischen Welt, der nanoskaligen Ebene der Atome und Moleküle ist ein potentiell unendliches Feld, das seine Konkretisierung im Sinne antizipierter Produkt- und Verfahrensinnovationen erst im politischen Raum erhält. In diesem verständigen sich Akteure aus Wissenschaft, Industrie und Investoren darüber, wo die Grenzen des Machbaren, die höchsten Potentiale und die viel versprechendsten Anwendungen liegen und wie die Risiken zu managen sind.

In der Governance der Wahrheit wird das technisch Mögliche der Nanotechnologie in einem steten Prozess verhandelt, temporär festgelegt und zugleich ein Prozess und ein Akteurskreis etabliert, der die Grenzen überprüft und gegebenenfalls anpasst. In diesem Prozess kristallisiert sich ein Kern der Technologie heraus, der unstrittig ist und der durch seine strittigen Grenzen laufend bestätigt wird. Die politische Ökonomie des Versprechens braucht beides: den unstrittigen Kern, der bereits kurz- bis mittelfristig auf tatsächliche Anwendungen und ein hohes Marktwachstum verweisen kann, aber auch die weder sichtbaren, noch erfahrbaren, aber langfristig machbar erscheinenden und als revolutionär klassifizierten Visionen, die radikale (Durch-)Brüche versprechen und damit hohe Risiken vertretbar erscheinen lassen. Die Faszination, die Nanotechnologie zum Gegenstand einer Ökonomie des Versprechens werden ließ, beruht gerade nicht auf den Produkt- und Verfahrensinnovationen, sondern auf dem Szenario einer neuen industriellen Revolution, deren Widerspruch eklatant ist, ohne jedoch als solcher thematisiert zu werden: Auf der einen Seite wird die Zukunft der Nanotechnologie als eine dargestellt, in der jeder alles haben kann, Knappheit zur Vergangenheit wird und die Annehmlichkeiten des modernen Lebens - für alle - mit dem Traum vom vormodernen Leben mit einer „intakten“ Natur zu verbinden ist. Für Banken und Investoren besteht die verlockende Perspektive wiederum gerade darin, Teile dieses Nanokosmos monopolisieren zu können. Gelänge es einem Unternehmen, „die Drexler'schen universellen Assembler herzustellen und eine effektive Technik zu ihrer Steuerung zu entwickeln, besäße es damit quasi die Lizenz zum Gelddrucken. Schlagartig hätte dieses Unternehmen die Möglichkeit zur Herstellung fast beliebiger Produkte." (HWAG/PERFORMAXX 2001: 25). 


\section{Literatur}

Allianz/OECD, Organisation for Economic Co-Operation and Development (2005): Small sizes that matter: Opportunities and risks of Nanotechnologies. Report in co-operation with the OECD International Futures Programme, Allianz Center for Technology, München; Paris.

Bachmann, Gerd (1994): Technologieanalyse Nanotechnologie. VDI Technologieanalyse, VDI, Düsseldorf.

Bachmann, Gerd (1998): Analyse und Bewertung zukünftiger Technologien. Innovationsschub aus dem Nanokosmos, VDI Technologiezentrum, Düsseldorf.

Baird, Davis/Shew, Ashley (2004): Probing the History of Scanning Tunneling Microscopy, in: Baird, Davis/Alfred Nordmann/Joachim Schummer (Hrsg.): Discovering the Nanoscale, Amsterdam: $145-156$.

BMBF, Bundesministerium für Bildung und Forschung (2002a): Standortbestimmung. Nanotechnologie in Deutschland, Bonn.

- (2002b): Strategische Neuausrichtung. Nanotechnologie in Deutschland, Bonn.

- (2004a): Nanotechnologie erobert Märkte. Deutsche Zukunftsoffensive für Nanotechnologie, Bonn.

- (2004b): NanoTruck. Reise in den Nanokosmos. Die Welt kleinster Dimensionen, Bonn.

- (2005): Nanotechnologie. Innovationen für die Welt von morgen, Bonn.

- (2006): Nano-Initiative - Aktionsplan 2010, BMBF, Bonn.

BMBF, Bundesministerium für Bildung, Wissenschaft, Forschung und Technologie (1998): Nanotechnologie. Innovationsschub aus dem Nanokosmos.

BMU Bundesministerium für Umwelt, Naturschutz und Reaktorsicherheit; Umweltbundesamt; Bundesanstalt für Arbeitsschutz und Arbeitsmedizin et al. (2005): Stakeholder Dialog Synthetische Nanopartikel. Dokumentation zur „Bewertung von synthetischen Nanopartikeln in Arbeits- und Umweltbereichen“, Dialog Nanopartikel, Bundesumweltministerium Bonn.

Bundesregierung (2006): Antwort der Bundesregierung auf die Kleine Anfrage der Abgeordneten Hans-Josef Fell, Ulrike Höfken, Priska Hinz (Herborn), weiterer Abgeordneter und der Fraktion BÜNDNIS 90/DIE GRÜNEN - Drucksache 16/2150 - Potenziale und Risiken der Nanotechnologie, Berlin.

Canzler, Weert; Dierkes, Meinolf (2001): Informationelle Techniksteuerung: Öffentliche Diskurse und Leitbildentwicklungen, in: Simonis, Georg; Renate Martinsen; Thomas Saretzki (Hrsg.): Politik und Technik. Analysen zum Verhältnis von technologischem, politischem und staatlichem Wandel am Anfang des 21. Jahrhunderts. PVS Sonderheft 31., Opladen: 457 - 475.

Coenen, Christopher (2004): Nanofuturismus: Anmerkungen zu seiner Relevanz, Analyse und Bewertung, in: Technikfolgenabschätzung - Theorie und Praxis 13 (2): 78-85.

Crichton, Michael (2002): Beute, München.

Drexler, Eric K.; Peterson, Chris; Pergamit, Gayle (1991): Experiment Zukunft. Die nanotechnologische Revolution, Bonn; Paris; Reading.

Drexler, K. Eric (1987): Engines of Creation. The Coming Era of Nanotechnology, New York.

Dürrenberger, Franz; Höck, Jürgen; Höhener, Franz (2004): Overview of completed an ongoing activities in the field: Safety and Risks in the Field of Nanotechnology, TEMAS AG, Arbon.

EEA, European Environment Agency (2001): Late lessons from early warnings. The precautionary principle 1896-2000, Copenhagen.

Eickenbusch, Heinz; Hoffknecht, Andreas; Holtmannspötter, Dirk et al. (2003): Technologiefrüherkennung. Monitoring-Bericht. Ansätze zur technischen Nutzung der Selbstorganisation., VDI Technologiezentrum GmbH, Düsseldorf.

Eigler, Don (1999): From the bottom up. Buildung things with Atoms, in: Timp, Gregory L. (Hrsg.): Nanotechnology, New York; Berlin; Heidelberg: 425-435.

Eigler, Don; Schweizer, E. K. (1990): Positioning single atoms with a scanning tunneling microscope, in: Nature 344 (5 April 1990): 524-526.

EPA, U.S. Environmental Protection Agency (2005): Nanotechnology White Paper. External Review Draft. Prepared for the U.S. Environmental Protection Agency by Members of the Nanotechnology Workgroup, a Group of EPA's Science Policy Council, Washington.

ETC Group (2002): No Small Matter! Nanotech Particles Penetrate Living Cells and Accumulate in Animal Organs, Winnipeg. 
ETC Group (2003a): From Genomes to Atoms. The Big Down. Atomtech: Technologies Converging at the Nano-scale, Winnipeg.

ETC Group (2003b): Green Goo: Nanobiotechnology Comes Alive!, Winnipeg.

ETC Group (2003c): Size Matters! The Case for a Global Moratorium. No Small Matter II, Winnipeg.

ETC Group (2004a): 26 Governments Tiptoe Toward Global Nano Governance. Grey-Goovernance? News Release, Winnipeg.

ETC Group (2004b): Down on the Farm: The Impact of Nano-scale Technologies on Food and Agriculture, Winnipeg.

ETC Group (2005): Nanotech's Second Nature' Patents: Implications for the Global South, Ottawa.

ETC Group (2006): Nanotech Rx. Medical Applications of Nano-scale Technologies: What Impact on Marginalized Communities?, Winnipeg.

Europäische Kommission (2004): Auf dem Weg zu einer europäischen Strategie für Nanotechnologie, Kommission der Europäischen Gemeinschaften, Brüssel.

European Commission (2004a): Towards a European Strategy for Nanotechnology, Commission of the European Communities, Brüssel.

European Commission (2005a): Communication from the Commission to the Council, the European Parliament and the Economic and Social Committee. Nanosciences and nanotechnologies: An action plan for Europe 2005-2009, Commission of the European Communities, Brussels.

European Commission (2005b): Social values, Science and Technology, Commission of the European Communities, Brüssel.

European Commission, Community Health and Consumer Protection Directorate General (2004b): Nanotechnologies: A Preliminary Risk Analysis On The Basis of a Workshop Organized in Brussels on 1-2 March 2004 by the Health and Consumer Protection Directorate General, Brüssel.

Feynman, Richard P. (1959): There's Plenty of Room at the Bottom. Vortrag am 29. Dezember 1959 (zuerst erschienen in: Engineering and Science), California Institute of Technology, in: http://www.its.caltech.edu/ feynman/plenty.html.

Foucault, Michel (1991): Die Ordnung des Diskurses, Frankfurt am Main.

Fraunhofer Institut für Silikatforschung/Innovationsforschung, Fraunhofer Institut für Systemtechnik und (2003): Produktion von und mit Nanomaterialien. Untersuchung des Forschungsund Handlungsbedarfs für die industrielle Produktion, Würzburg; Karlsruhe.

Gaskell, George; Einsiedel, Edna; Hallman, William et al. (2005): Communication: Enhanced: Social Values and the Governance of Science, in: Science 310 (5756): 1908-1909.

Gaskell, George; Eyck, Toby Ten; Jackson, Jonathan, et al. (2005): Imagining nanotechnology: cultural support for technological innovation in Europe and the United States, in: Public Understanding of Science 14 (1): 81-90.

Gieryn, Thomas F (1995): Boundaries of Science, in: Jasanoff, Sheila; Gerald E. Markle; James C. Petersen; Trevor C. Pinch (Hrsg.): Handbook of Science and Technology Studies, Thousand Oakes: 393-444.

Gieryn, Thomas F (1999): Cultural Boundaries of Science, Chicago.

Gottweis, Herbert (1998): Governing Molecules. The Discursive Politics of Genetic Engineering in Europe and the United States, Cambridge, Massachusetts; London, England.

Gottweis, Herbert (2005): Governing Genomics in the 21st century: Between Risk and Uncertainty, in: New Genetics \& Society 24 (2): 175-194.

Grande, Edgar (2001): Von der Technologiepolitik zur Innovationspolitik. Europäische Forschungs- und Technologiepolitik im Zeitalter der Globalisierung, in: Simonis, Georg; Renate Martinsen; Thomas Saretzki (Hrsg.): Politik und Technik. Analysen zum Verhältnis von technologischem, politischem und staatlichem Wandel am Anfang des 21. Jahrhunderts, Opladen: 368-387.

Greenpeace Magazin (2000).

Grobe, Antje; Eberhard, Caspar; Hutterli, Martin (2005): Nanotechnologie im Spiegel der Medien: Medienanalyse zur Berichterstattung über Chancen und Risiken der Nanotechnologie. Januar 2001 - April 2005, Stiftung Risiko-Dialog, St. Gallen.

Grunwald, Armin (2004): Vision Assessment as New Element of the FTA Toolbox. Vortrag, EU-US Seminar: New Technology Foresight, Forecasting \& Assessment Methods, 13.-14.05.2004, Sevilla. 
Haraway, Donna (1997): Modest_Witness@Second_Millenium. FemaleMan_Meets_OncoMouse, London.

Helmer, Olaf; Gordon, Theodore J. (1967): 50 Jahre Zukunft. Bericht über eine LangfristVorhersage für die Welt der nächsten fünf Jahrzehnte, Hamburg.

HWAG, Hanseatisches Wertpapierhandelshaus/PERFORMAXX (2001): Research-Studie. Nanotechnologie I. Grundlagen., Hamburg.

International Risk Governance Council (2004): Nanotechnology, Geneva.

Joly, Pierre-Benoit; Callon, Michel; Dianoux, Laurent et al. (2005): Democratie locale et Maitrise sociale des Nanotechnologies, http://sciencescitoyennes.org/IMG/pdf/NanoGrenoble rapport_final_05_09_22.pdf.

Joy, Bill (2000a): Warum die Zukunft uns nicht braucht. Die mächtigsten Technologien des 21. Jahrhunderts - Robotik, Gentechnik und Nanotechnologie - machen den Menschen zur gefährdeten Art, in: Frankfurter Allgemeine Zeitung, 130, 6. Juni: 49-51.

Joy, Bill (2000b): Why the future doesn't need us. Our most powerful 21st-century technologies - robotics, genetic engineering, and nanotech - are threatening to make humans an endangered species., in: Wired (Issue 8.04).

Kahn, Herman; Wiener, Anthony J. (1971): Ihr werdet es erleben. Voraussagen der Wissenschaft bis zum Jahre 2000, Reinbek bei Hamburg.

Kurath, Monika; Maasen, Sabine (2006): Toxicology as a Nanoscience? - Disciplinary Identities Reconsidered, in: Particle and Fibre Toxicology 3 (6): o. S.

Lee, Chul-Joo; Scheufele, Dietram A.; Lewenstein, Bruce V. (2005): Public Attitudes toward Emerging Technologies: Examining the Interactive Effects of Cognitions and Affect on Public Attitudes toward Nanotechnology, in: Science Communication 27 (2): 240-267.

Luther, Wolfgang (2004): Industrial application of nanomaterials - chances and risks. Technology analysis., VDI-Technologiezentrum GmbH, Düsseldorf.

Luther, Wolfgang; Bachmann, Gerd; Zweck, Axel et al. (2006): Kommerzialisierung der Nanotechnologie, VDI Technologiezentrum, Düsseldorf.

Luther, Wolfgang; Malanowski, Norbert; Bachmann, Gerd et al. (2004): Nanotechnologie als wirtschaftlicher Wachstumsmarkt, VDI-Technologiezentrum, Düsseldorf.

Malsch, Ineke; Oud, Mireille (2004): Outcome of the Open Consultation on the European Strategy for Nanotechnology, nanoforum.org, o. O.

Marcinkowski, Frank (2001): Öffentliche Kommunikation als präventive Risikoerzeugung - Politikwissenschaftlich relevante Ansätze der Risikoforschung und neue empirische Befunde, in: Simonis, Georg; Renate Martinsen; Thomas Saretzki (Hrsg.): Politik und Technik. Analysen zum Verhältnis von technologischem, politischem und staatlichem Wandel am Anfang des 21. Jahrhunderts: 147-166.

Marlow, John Robert (2005): Nano, New York.

Milburn, Colin (2002): Nanotechnology in the Age of Posthuman Engineering: Science Fiction as Science, in: Configurations 10 (2, Spring 2002): 261-296.

Nanoforum Consortium (2004): 4th Nanoforum Report: Benefits, Risks, Ethical, Legal and Social Aspects of Nanotechnology, nanoforum.org, o. O.

National Science and Technology Council (1999): Nanotechnology. Shaping the World Atom by Atom, Washington.

National Science and Technology Council (2004): National Nanotechnology Initiative. Strategic Plan, Washington.

Nordmann, Alfred (2003): Shaping the World Atom by Atom: Eine nanowissenschaftliche WeltBildanalyse, in: www.cla.sc.edu/cpecs/nirt/papers/AN2.html.

President's Council of Advisors on Science and Technology (2005): The National Nanotechnology Initiative at Five Years: Assessment and Recommendations of the National Nanotechnology Advisory Panel, Washington D. C.

Roco, Mihail C. (2004): The US National Nanotechnology Initiative after 3 years (2001-2003), in: Journal of Nanoparticle Research (6): 1-10.

Roco, Mihail C. (2005): Environmentally responsible development of nanotechnology. How the U.S. Government is dealing with the Immediate and Long-term Issues of this New Technology, in: Environmental Science \& Technology (1): 107- 112. 
Roco, Mihail C.; Bainbridge, William Sims (2002): Converging Technologies for Improving Human Performance. Nanotechnology, Biotechnology, Information Technology and Cognitive Science.

Royal Society (2005): Government commits to regulating nanotechnologies but will it deliver?, in: http://www.royalsoc.ac.uk/news.asp?id=2976.

Royal Society; The Royal Academy of Engineering (2004): Nanoscience and nanotechnologies: opportunities and uncertainties, London.

Saretzki, Thomas (2001): Entstehung, Verlauf und Wirkungen von Technisierungskonflikten: Die Rolle von Bürgerinitiativen, sozialen Bewegungen und politischen Parteien, in: Simonis, Georg; Renate Martinsen; Thomas Saretzki (Hrsg.): Politik und Technik. Analysen zum Verhältnis von technologischem, politischem und staatlichem Wandel am Anfang des 21. Jahrhunderts: $185-210$.

Saretzki, Thomas (2005): Welches Wissen - Welche Entscheidung? Kontroverse Expertise im Spannungsfeld von Wissenschaft, Öffentlichkeit und Politik, in: Bogner, Alexander; Helge Torgersen (Hrsg.): Wozu Experten? Ambivalenzen in der Beziehung von Wissenschaft und Politik, Wiesbaden: 345-369.

Schaper-Rinkel, Petra (2006a): Nanotechnologiepolitik: Die Antizipation potentieller Umweltund Technikkonflikte in der Governance der Nanotechnologie, in: Feindt, Peter H.; Thomas Saretzki (Hrsg.): Umwelt- und Technikkonflikte, Wiesbaden.

Schaper-Rinkel, Petra (2006b): Politik als Initiierung von Spielfeldern und Setzung von Spielregeln. Forcierung und Regulierung der Nanotechnologie, in: Gebauer, Gunter; Stefan Poser; Robert Schmidt; Martin Stern (Hrsg.): Kalkuliertes Risiko, Frankfurt am Main; New York.

Steinfeldt, Michael; Gleich, Arnim von; Petschow, Ulrich et al. (2004): Nachhaltigkeitseffekte durch Herstellung und Anwendung nanotechnologischer Produkte, Institut für ökologische Wirtschaftsforschung gGmbH, Berlin.

Stephens, Lowndes F. (2005): News Narratives about Nano S\&T in Major U.S. and Non-U.S. Newspapers, in: Science Communication 27 (2): 175-199.

Stephenson, Neal (1996): Diamond Age. Die Grenzwelt, München.

Swiss Re, Swiss Reinsurance Company (2004a): Nanotechnologie. Kleine Teile - große Zukunft, Zürich.

Swiss Re, Swiss Reinsurance Company (2004b): Nanotechnology. Small matter, many unknowns, Zürich.

TAB, Büro für Technikfolgen-Abschätzung beim Deutschen Bundestag (2003): TA-Projekt Nanotechnologie. Endbericht, Büro für Technikfolgenabschätzung beim Deutschen Bundestag, Berlin; Karlsruhe.

TAB, Büro für Technikfolgen-Abschätzung beim Deutschen Bundestag (2004): Bericht des Ausschusses für Bildung, Forschung und Technikfolgenabschätzung (17. Ausschuss) gemäß § 56a der Geschäftsordnung Technikfolgenabschätzung hier: TA-Projekt - Nanotechnologie. Drucksache 15/2713, Berlin.

Taniguchi, Norio (1974): On the Basic Concept of 'Nano-Technology', in: Bulletin of the Japan Society of Precision Engineering: 18-23.

UK Government (2005): Response to the Royal Society and Royal Academy of Engineering Report. 'Nanoscience and nanotechnologies: opportunities and uncertainties', HM Government.

VDI Technologiezentrum (o. J.-a): The Journey into the nano-cosmos. Presentation materials. Part I: Scientific and technical principles. o. O.

VDI Technologiezentrum (o. J.-b): The Journey into the nano-cosmos. Presentation materials. Part III: Social and Political Aspects. o. O.

VDI Technologiezentrum (o. J.-c): Reise in den Nanokosmos. Teil I: Wissenschaftlich-technische Grundlagen. Präsentationsmaterial. o. O.

Wagner, Volker; Wechsler, Dietmar (2004): Nanobiotechnologie II. Anwendungen in der Medizin und Pharmazie, VDI-TEchnologiezentrum, Düsseldorf. 